\title{
Uma Medida de Satisfação com o Relacionamento Amoroso
}

\author{
Amanda Londero-Santos ${ }^{1}$ (D) \\ Universidade Federal do Rio de Janeiro, Rio de Janeiro-RJ, Brasil \\ Jean Carlos Natividade (D), Terezinha Féres-Carneiro (D) \\ Pontifícia Universidade Católica do Rio de Janeiro, Rio de Janeiro-RJ, Brasil
}

\section{RESUMO}

Esta pesquisa teve por objetivo adaptar para o contexto brasileiro a Escala do Nível de Satisfação com o Relacionamento Amoroso (ENSRA, Rusbult, Martz, \& Agnew, 1998). No primeiro estudo ( $N=269)$, buscaram-se evidências de validade baseadas na estrutura unifatorial do instrumento e analisaram-se as propriedades dos itens de acordo com a Teoria de Resposta ao Item. No segundo estudo $(N=1.498)$, propôs-se uma versão revisada da escala (ENSRA-R), incluindo-se novos itens com parâmetros mais elevados de dificuldade. Análises fatoriais exploratórias e confirmatórias indicaram uma estrutura unifatorial também para ENSRA-R. Conforme esperado teoricamente, ENSRA e ENSRA-R correlacionaram-se positivamente com outra medida de satisfação com o relacionamento e com amor romântico, e negativamente com intenção de terminar o relacionamento. Ambas as versões do instrumento apresentam satisfatórias evidências de validade e adequados índices de precisão, contudo, a ENSRA-R mostrou-se mais informativa, cobrindo uma maior porção do traço latente, comparada à ENSRA.

Palavras-chave: satisfação com o relacionamento amoroso; adaptação de escala; amor.

\section{ABSTRACT - A Measure of Satisfaction in the Romantic Relationship}

This study aimed to adapt the Investment Model of Commitment Processes Scale (Escala do Nível de Satisfação com o Relacionamento Amoroso - ENSRA - Rusbult, Martz, \& Agnew, 1988) to the Brazilian context. In the first study $(N=269)$, we sought evidence of validity based on the unifactorial structure of the instrument and analyzed the properties of the items according to the item response theory. In the second study $(N=1498)$, a revised version of the scale (ENSRA-R) was proposed, including new items with higher difficulty parameters. Exploratory and confirmatory factor analyses indicated a single-factor structure for both the ENSRA and ENSRA-R. As theoretically expected the ENSRA and ENSRA-R correlated positively with another instrument that assesses relationship satisfaction and with romantic love, and negatively with the intention to break-up. Both versions of the instrument provide satisfactory evidence of validity and adequate accuracy, however, the ENSRA-R was more informative, covering a larger portion of the latent trait compared to the ENSRA.

Keywords: relationship satisfaction; cross cultural test adaptation; love.

\section{RESUMEN - Una Escala de Satisfacción en la Pareja}

Esta investigación tuvo como objetivo adaptar la Escala de Satisfacción en la Pareja (ENSRA, Rusbult, Martz, \& Agnew, 1988) al contexto brasileño. En el primer estudio $(N=269)$, se buscaron evidencias de validez basadas en la estructura unifactorial del instrumento y, de acuerdo con la teoría de respuesta al ítem, se analizaron las propiedades de los ítems. En el segundo estudio (N $=1498$ ) fue propuesta una versión revisada de la escala (ENSRA-R), que incluye nuevos ítems con parámetros de dificultad más estrictos. Los análisis factoriales exploratorios y confirmatorios también indicaron la estructura de un factor para la ENSRA-R. Como resultado esperado, ENSRA y ENSRA-R se correlacionaron positivamente con otra medida de satisfacción con la relación y con el amor romántico; y negativamente con la intención de terminar la relación. Ambas versiones del instrumento presentan evidencias de validez satisfactorias e índices de precisión adecuados. Sin embargo, la ENSRA-R se demostró más informativa, cubriendo el rasgo latente en mayor medida, en comparación con la ENSRA.

Palabras clave: satisfacción en la pareja; adaptación de test; amor.

Satisfação com o relacionamento amoroso é um dos principais temas estudados na área de família e casal e de relacionamentos. A satisfação com o relacionamento tem impacto em diversos desfechos positivos, por exemplo, na persistência e manutenção da relação amorosa (e.g., Rusbult et al., 2011; Weiser \& Weiser, 2016), na satisfação

\footnotetext{
${ }^{1}$ Endereço para correspondência: Pontifícia Universidade Católica do Rio de Janeiro. Rua Marquês de São Vicente, 225, Gávea, 22451-900, Rio de Janeiro, RJ Tel.: (21) 3527-2080.E-mail: amandalondero@me.com

Artigo derivado da Tese de doutorado de Amanda Londero-Santos com orientação da Profa. Terezinha Féres-Carneiro, defendida em 2020 no Programa de Pós-Graduação em Psicologia da Pontifícia Universidade Católica do Rio de Janeiro (PUC-Rio)

O presente trabalho foi realizado com apoio do Conselho Nacional de Desenvolvimento Científico e Tecnológico (CNPq), da Fundação de Amparo à Pesquisa do Estado do Rio de Janeiro - FAPERJ, e da Coordenação de Aperfeiçoamento de Pessoal de Nível Superior - Brasil (CAPES).
} 
sexual com o parceiro (e.g., McNulty et al., 2016), na saúde e bem-estar (e.g., Hollist et al., 2016; Londero-Santos et al., 2017; Scorsolini-Comin \& Santos, 2011). Apesar da relevância desse tema, poucos instrumentos para avaliar a satisfação global com o relacionamento amoroso apresentam satisfatórias evidências de validade e fidedignidade para população brasileira. Esta pesquisa teve o objetivo de adaptar para o contexto brasileiro a Escala do Nível de Satisfação com o Relacionamento Amoroso, desenvolvida por Rusbult et al. (1988), uma escala mundialmente utilizada para avaliar a satisfação com o relacionamento amoroso, e buscar evidências de validade e precisão.

Diversas definições de satisfação com o relacionamento amoroso foram propostas na literatura científica. Muitas destas consideram a satisfação como um balanço entre aspectos positivos e negativos da relação amorosa (e.g., Shackelford \& Buss, 1997; Wachelke et al., 2004). Para Rusbult (1980), o julgamento sobre a satisfação com o relacionamento depende da comparação de benefícios e recompensas ligadas ao relacionamento com as expectativas sobre o relacionamento. A satisfação também pode ser considerada uma atitude frente ao relacionamento com o parceiro amoroso (Londero-Santos et al., 2018; Roach et al., 1981). Assim, ela refere-se a uma associação entre o conceito "relacionamento" e a "avaliação sobre esse conceito" (para uma revisão sobre atitude e esquema social, ver Briñol et al., 2019; Greenwald et al., 2002; Greifeneder et al., 2018).

Os instrumentos que visam a mensurar a satisfação com o relacionamento podem ser classificados, quanto à sua estrutura fatorial, como unidimensionais ou multidimensionais (para um levantamento dos instrumentos existentes para avaliar construtos relacionados a relacionamentos românticos disponíveis em língua portuguesa, ver Cassepp-Borges \& De Andrade, 2013). Dentre as escalas multidimensionais com evidências de validade para o Brasil, podem-se citar a Escala de Satisfação Conjugal (ESC, desenvolvida por Pick de Weiss \& Andrade Palos, 1988, adaptada para o contexto brasileiro por Dela Coleta, 1989; e revisada por Hernandez et al., 2017) e a escala de satisfação com o relacionamento de casal (Wachelke et al., 2004). Quanto aos instrumentos unidimensionais com evidências de validade para o Brasil, tem-se conhecimento apenas da Relationship Assessment Scale (RelAS, adaptada para o contexto brasileiro por Cassepp-Borges \& Pasquali, 2011 e por Hernandez, 2014).

Além desses três instrumentos, existem outros com evidências de validade para o Brasil que medem construtos associados à satisfação com relacionamentos amorosos. Por exemplo, a subescala de satisfação diádica da Escala de Ajustamento Diádico (DAS, adaptada para o Brasil por Hernandez, 2008), a Escala Amor que afere o vínculo emocional do Marriage and Relationships Questionnaire (adaptada para o Brasil por França et al., 2016), a Escala de Avaliação da Qualidade em Relacionamentos Românticos (AQUARELA-R, De
Andrade \& Garcia, 2012). No entanto, essas escalas, apesar de terem propriedades psicométricas adequadas, apresentam dificuldades para a avalição da satisfação com relacionamentos amorosos. A subescala da DAS que avalia a satisfação conjugal, por exemplo, inclui itens que são aplicáveis apenas a relacionamentos maritais (e.g., "Arrepender-se de ter casado"). Já a Escala Amor e a AQUARELA-R não mensuram especificamente a satisfação com o relacionamento. Ainda, há instrumentos que foram traduzidos ou construídos ad-hoc e utilizados no Brasil para aferir satisfação com o relacionamento (e.g., De Andrade et al., 2017; Hollist et al., 2016; Miranda, 1987), no entanto, desconhecem-se estudos que apresentem suas evidências de validade e precisão.

Essa diversidade de instrumentos, cada qual com fundamentação teórica diferente, traz dificuldade para comparar achados empíricos. Scorsolini-Comin e Santos (2011), por exemplo, investigaram a relação entre a subescala Satisfação Diádica da DAS e os subfatores da Escala de Satisfação Conjugal (ESC) e encontraram coeficientes de correlações entre 0,28 a 0,31 . Esses resultados demonstram que a quantidade de variância compartilhada entre essas escalas é pequena. Isso sugere que essas medidas, embora sejam usadas para acessar a satisfação com o relacionamento, não medem o mesmo construto.

Além disso, ainda que as medidas multidimensionais sejam importantes, pois permitem acessar as dimensões que são potencialmente utilizadas para avaliar a satisfação global com o relacionamento, não representam adequadamente uma satisfação global com o relacionamento. Avaliar satisfação com o relacionamento por meio de aspectos específicos corresponde a avaliar atitudes frente a conceitos específicos associados ao relacionamento com o parceiro amoroso (e.g., vida sexual, planos futuros). No entanto, essas avaliações não permitem conhecer a atitude específica frente ao relacionamento com o parceiro amoroso (i.e., a satisfação global com o relacionamento amoroso). Nesse sentido, mesmo considerando que a satisfação global com o relacionamento derive de satisfações de aspectos específicos do relacionamento (e.g., satisfação com atração física e sexualidade), instrumentos multidimensionais podem valorizar excessivamente, ou mesmo deixar de considerar, aspectos que os indivíduos incluem nos seus esquemas de relacionamento positivo (ver Londero-Santos et al., 2018). Não é possível afirmar, portanto, em quais aspectos específicos do relacionamento uma pessoa se baseia para chegar à avaliação geral sobre seu relacionamento.

Por exemplo, a Escala de Satisfação com o Relacionamento de Casal de Wachelke et al. (2004) avalia dois aspectos da satisfação: 1. satisfação com atração física e sexualidade; 2 . satisfação com afinidades de interesses e comportamentos. A sexualidade pode ser um aspecto específico que contribui para a satisfação global com o relacionamento, no entanto, outros aspectos também podem contribuir para essa avaliação global (e.g., satisfação com as divisões de tarefa entre o casal). Assim, os dois aspectos 
avaliados na escala de Wachelke et al. podem, para muitos indivíduos, não ser os mais importantes para a avaliação geral do seu relacionamento amoroso. Evidências, de fato, sugerem que a sexualidade em relacionamento de longo prazo de pessoas idosas não tem um impacto significativo na satisfação global com o relacionamento amoroso (e.g., Müller et al., 2014). Assim, inferir uma avaliação global sobre a satisfação com o relacionamento a partir de avaliações de domínios específicos pode levar a resultados imprecisos, já que o construto geral poderia incluir outros aspectos que não foram levados em consideração pelas escalas específicas.

A Relationship Assessment Scale (RelAS, CasseppBorges \& Pasquali, 2011; Hendrick, 1998; Hernandez, 2014) afere a satisfação global com o relacionamento. Trata-se da única escala unidimensional com evidências de validade e fidedignidade para população brasileira que se tem conhecimento. No contexto internacional, outro instrumento unidimensional muito utilizado para medir satisfação com o relacionamento é a Escala do Nível de Satisfação com o Relacionamento Amoroso desenvolvida por Rusbult et al. (1998). Essa escala tem sido utilizada em diversos países (e.g., Amirsardari \& Khademi, 2019; Gable \& Poore, 2008; Hoff et al., 2019; Joel et al., 2013; Rodrigues \& Lopes, 2013; Suntornkanit \& Varma, 2018; Tan et al., 2018; VanderDrift et al., 2014; Wickham, 2013; Wickham et al., 2015; Winking et al., 2018). Uma versão dela para o Brasil incrementaria a comparabilidade dos resultados das pesquisas nacionais.

A Escala do Nível de Satisfação com o Relacionamento Amoroso (ENSRA) foi desenvolvida de acordo com o modelo de investimento do processo de comprometimento e é parte de uma bateria maior que avalia também qualidade de alternativas ao relacionamento, a quantidade de investimento no relacionamento e o nível de comprometimento com o relacionamento amoroso (Rusbult et al., 1998, para uma revisão, ver Tran, et al., 2019). As escalas que compõem a bateria do modelo de investimento do processo de comprometimento são independentes entre si. De fato, a ENSRA tem sido utilizada de forma independente das outras escalas que fazem parte da bateria (e.g., Gable \& Poore, 2008; Hoff et al., 2019; Impett et al., 2005; Joel et al., 2013; Wickham et al., 2015). Os itens da ENSRA são de dois tipos: cinco itens aparentes (de face), que perguntam sobre exemplos concretos do relacionamento (e.g., "Meu parceiro satisfaz minhas necessidades de intimidade..."), e cinco itens sobre aspectos gerais do relacionamento (e.g., "Eu me sinto satisfeito com o nosso relacionamento"). As respostas aos itens aparentes não são usadas para aferir o construto, eles são usados apenas aumentar a compreensão dos itens sobre aspectos gerais.

A análise fatorial dos itens da ENSRA evidenciou uma estrutura unidimensional, não apresentando carga fatorial cruzada com as demais escalas presentes na bateria (Rusbult et al., 1998). A ENSRA apresentou adequados indicadores de fidedignidade e evidências de validade do tipo concorrente (e.g., satisfação e ajustamento diádico, amor pelo parceiro) e divergente (e.g., qualidade de alternativas ao relacionamento, necessidade de independência) (Rusbult et al. 1998). O instrumento ainda apresenta uma baixa correlação com desejabilidade social (correlações com os fatores da desejabilidade social, variando entre 0,13 e 0,15). Além disso, a escala conseguiu distinguir indivíduos que persistiram e terminaram seus relacionamentos. Foi observada diferença significativa do escore na ENSRA entre aqueles que continuaram e aqueles que romperam seus relacionamentos (Rusbult et al., 1998).

A ENSRA tem sido utilizada em várias pesquisas para analisar aspectos dos relacionamentos amorosos. O instrumento tem se mostrado útil para predizer comprometimento com o relacionamento amoroso (Rhatigan \& Axsom, 2006; Rusbult et al., 1998) e dissolução dos relacionamentos (Rusbult et al., 1998; VanderDrift et al., 2014), e relações com: sacrifícios nos relacionamentos íntimos (Impett et al., 2005), autenticidade nos relacionamentos (Wickham et al., 2015), relacionamentos abusivos (Rhatigan \& Axsom, 2006), violência nos relacionamentos (Overup et al., 2017) e sentido de vida (Hadden \& Knee, 2018).

Diante da pouca disponibilidade de instrumentos breves no Brasil para aferir a satisfação global com o relacionamento, delineou-se esta pesquisa com o objetivo de adaptar para o contexto brasileiro a Escala do Nível de Satisfação com o Relacionamento de Rusbult et al. (1998). Para atingir o objetivo, foram desenvolvidos dois estudos independentes. O primeiro foi um estudo piloto, conforme sugere o Guideline da International Teste Commission (2017), no qual (1) buscaram-se evidências de fidedignidade e de validade da escala, e (2) analisaram-se as propriedades dos itens de acordo com a Teoria de Resposta ao Item. O segundo estudo propôs uma versão revisada da escala, no qual (1) procuraram-se evidência de validade e fidedignidade para a versão original e revisada, (2) verificou-se a invariância de medida das escalas e (3) investigaram-se diferenças de gênero e orientação sexual nos níveis de satisfação com o relacionamento amoroso.

\section{Estudo 1}

\section{Método}

\section{Participantes}

Participaram do estudo 269 adultos que estavam em um relacionamento amoroso, sendo 214 mulheres (79,6\%). A média de idade foi de 35,4 anos $(D P=10,6)$, mínimo de 19 e máximo de 84 anos. A maioria era heterossexual $(90,7 \%)$, os demais eram homossexuais $(4,46 \%)$ e bissexuais (4,83\%). Quanto ao nível de escolaridade, $67,2 \%$ responderam estar frequentando ou ter concluído 
curso de pós-graduação; 28,7\% responderam estar frequentando ou ter frequentado ensino superior; $4,10 \%$ frequentaram ensino médio ou ensino fundamental. A maior parte dos participantes residia nas regiões sudeste $(69,5 \%)$ e sul $(20,4 \%)$ do Brasil, os demais eram da região centro-oeste $(4,46 \%)$, nordeste $(4,09 \%)$ e de fora do Brasil (1,57\%). No que diz respeito ao relacionamento amoroso, a maioria declarou viver junto com o parceiro $(93,7 \%)$. O tempo médio de relacionamento foi de 9,24 anos $(D P=9,35)$. Pouco menos da metade dos participantes, $133(49,4 \%)$, tinham filhos.

\section{Instrumentos}

Os dados foram levantados por meio de um questionário on-line, disponibilizado na internet, contendo questões sociodemográficas (e.g., gênero, orientação sexual, escolaridade, tempo de relacionamento) e a Escala do Nível de Satisfação com o Relacionamento Amoroso (ENSRA, Rusbult et al., 1998).

Escala do Nível de Satisfação com o Relacionamento Amoroso (ENSRA, Rusbult et al., 1998). Tratase de uma escala adaptada para o Brasil neste estudo. O instrumento contém cinco itens em formato de afirmativas para serem respondidos numa escala de concordância de nove pontos, tal que $0=$ Discordo completamente e $8=$ Concordo completamente. Quanto maiores os escores, maior a satisfação com o relacionamento amoroso. Na versão original, a escala apresentou coeficiente alfa de 0,95 .

\section{Procedimentos de Tradução}

Duas pessoas bilíngues (inglês-português), independentemente, traduziram a ENSRA do inglês para o português. Em seguida, uma terceira pessoa bilíngue, com experiência em adaptação de instrumentos psicológicos, comparou as versões traduzidas com o original em inglês e compilou as traduções em uma versão em português. Apresentou-se, então, a versão compilada a três pessoas da população geral para que julgassem a adequação da redação dos itens. A partir disso, fizeram-se pequenos ajustes de redação e elaborou-se a versão do instrumento para ser posta à prova empírica.

\section{Procedimentos de Coleta de Dados}

Os participantes foram recrutados por meio de convites por e-mail e em redes sociais na internet. Aqueles que aceitavam participar deviam clicar no link do endereço disponibilizado no convite e eram direcionados para o questionário. A pesquisa foi encaminhada ao Comitê de Ética em Pesquisa com Seres Humanos e obteve parecer favorável.

\section{Procedimentos de Análise de Dados}

Após exclusão de casos omissos nos itens da ENSRA, procederam-se análises dos dados com o software R (R Core Team, 2018). A fim de testar o ajuste dos dados ao modelo unidimensional proposto para o instrumento, realizou-se análise fatorial confirmatória para dados ordinais, partindo-se da matriz de correlação policórica, utilizando-se o pacote Lavaan. A fim de decidir sobre o estimador para os índices de ajuste, primeiramente, verificou-se a normalidade multivariada dos dados por meio do teste de Mardia, utilizando o pacote semTools. Para estimar os parâmetros dos itens, utilizou-se o Modelo de Resposta Gradual da Teoria de Resposta ao Item (Graded Response Model - GRM; Samejima, 1969) e o pacote Mirt.

\section{Resultados}

O coeficiente de curtose multivariada estandardizado de Mardia indicou ausência de normalidade multivariada dos dados $(z=33,1 ; p<0,001)$. O estimador empregado para a análise fatorial confirmatória foi o Diagonally Weighted Least Squares (DWLS), tendo em vista que esse estimador é adequado e frequentemente utilizado para analisar variáveis não normais e ordinais (Vecchione et al., 2013). Os índices de ajuste dos dados ao modelo unifatorial foram os seguintes: $\chi^{2}=4,06 ; g l=5$; $p=0,54 ; \chi^{2} / g l=0,81, \mathrm{CFI}=1,00, \mathrm{TLI}=1,00$, RMSEA $<$ 0,01 (IC $90 \%=0,00-0,08$ ). O valor do coeficiente alfa de Cronbach da escala foi de 0,91 e o ômega de 0,90. Procedeu-se, então, a análise dos itens por meio do GRM. Os parâmetros de discriminação dos itens da escala variaram de 1,31 a 8,88 ; já os thresholds variaram de $-3,04$ a 1,09 .

De acordo com classificação de Baker (2001), os itens da ENSRA apresentaram parâmetros a muito altos $(a>1,70)$, com exceção do item 2 , que apresentou nível moderado $(a=1,31)$. A análise dos thresholds dos itens indicou ausência de itens capazes de estimar adequadamente altos níveis do traço latente (alta satisfação com o relacionamento). Considera-se necessário, para uma estimação mais precisa dos níveis mais altos do traço latente, incluírem-se no instrumento itens com thresholds mais elevados. Diante disso, criaram-se quatro itens com o intuito de aumentar a precisão do teste nos níveis mais elevados de satisfação com o relacionamento, chegando, assim, a uma versão Beta da ENSRA. Foi realizado o Estudo 2 para verificar as propriedades psicométricas dessa versão.

\section{Estudo 2}

\section{Método}

\section{Participantes}

Participaram 1.498 pessoas provenientes das cinco regiões do Brasil, com média de idade de 33,4 $(D P=9,71)$, variando de 18 a 72 anos, $64,3 \%$ eram mulheres. A maioria dos participantes declarou-se heterossexual $(85,8 \%)$, os demais, homossexuais $(6,70 \%)$ e 
bissexuais (7,50\%). Quanto à escolaridade, a maioria dos participantes frequentava ou havia realizado curso de pós-graduação (63,0\%), particularmente, especialização $(24,9 \%)$, mestrado $(21,13 \%)$ e doutorado $(16,8 \%)$. Os demais estavam cursando, ou haviam concluído, ensino superior $(33,8 \%)$, ou tinham até ensino médio completo $(3,30 \%)$. A maior parte dos participantes residia na região Sudeste $(39,4 \%)$ e na região Sul $(37,8 \%)$; na região Nordeste residiam 11,6\% dos participantes; na Centro-Oeste, $5,40 \%$; na região Norte, $3,20 \%$; os demais $(2,70 \%)$ residiam fora do Brasil. Todos os participantes declararam estar envolvidos em relacionamento amoroso compromissado (e.g., namorando, noivo, casado, morando junto), a maioria (66,7\%) estava vivendo junto com seu parceiro. O tempo médio de relacionamento foi de 8,70 anos $(D P=8,66)$. A maioria dos participantes $(67,6 \%)$ não tinha filhos.

\section{Instrumentos}

Utilizou-se um questionário on-line, disponibilizado na internet, contendo questões sociodemográficas (e.g., gênero, tempo de relacionamento) e as escalas descritas abaixo.

Escala do Nível de Satisfação com o Relacionamento Amoroso - Beta (ENSRA-Beta). Com o objetivo de incluir itens com parâmetros $b$ mais elevados, a escala ENSRA, descrita no Estudo 1 foi acrescida de quatro itens, a saber: item $6=$ "Nosso relacionamento é perfeito"; item 7 = "Não mudaria nada em nosso relacionamento"; item 8 = "Nosso relacionamento tem aspectos negativos"; item $9=$ "Acredito que estarei satisfeito com nosso relacionamento amoroso no futuro".

Relationship Assessment Scale (RelAS; CasseppBorges \& Pasquali, 2011). O instrumento, traduzido e adaptado para o Brasil do original (Hendrick, 1988), é uma medida unidimensional que afere níveis de satisfação no relacionamento. Ela é composta por sete itens respondidos em uma escala de intensidade de sete pontos, sendo que 1 representa baixa intensidade (e.g., "nada") e 7, alta intensidade (e.g., "totalmente"). Exemplos de item: "O quanto você ama seu(sua) parceiro(a)?". A versão brasileira do instrumento apresentou coeficiente alfa igual a 0,85 (Cassepp-Borges \& Pasquali, 2011).

Escala Tetrangular do Amor (ETA; Gouveia et al., 2013). ETA é uma versão modificada da Escala Triangular do Amor (Sternberg, 1997) por Yela (2006). Ela foi traduzida e adaptada para o Brasil por Gouveia et al. (2013) e afere o amor a partir de quatro componentes: paixão erótica, paixão romântica, intimidade, compromisso. Para responder aos itens, o participante deve preencher mentalmente um espaço em branco com o nome do seu parceiro amoroso. Cada componente é composto por cinco itens, respondidos em uma escala Likert, variando de 1 ("Discordo totalmente") a 5 ("Concordo totalmen$t e$ ”). Os coeficientes alfas variaram de 0,74 a 0,92 .
Além disso, no questionário, havia uma pergunta para investigar a intenção de terminar o relacionamento para ser respondida em uma escala de frequência de sete pontos, variando de $0=$ Nunca a $6=$ Todos os dias ("Com qual frequência você pensa em terminar o seu relacionamento amoroso?”). Enfim, junto aos itens da ENSRA-Beta, incluiu-se uma questão de controle em que se solicitava ao participante responder de uma maneira específica (e.g., "Esta é apenas uma pergunta de controle, por favor, marque o número quatro"). Esse item foi usado para excluir possíveis respostas falsas. O questionário foi configurado de modo a não permitir respostas omissas aos itens das escalas.

\section{Procedimentos}

Os procedimentos de coleta foram iguais ao do Estudo 1.

\section{Análise de Dados}

Inicialmente, realizou-se uma limpeza dos dados, excluindo os participantes com respostas incorretas da questão controle. Tendo em vista a inclusão de quatro novos itens na versão beta da escala, realizou-se uma análise fatorial exploratória (AFE) para investigar sua estrutura fatorial. Para tanto, a amostra foi dividida aleatoriamente em duas metades, uma para a análise fatorial exploratória (AFE, $n=772$ ) e outra para análise fatorial confirmatória (AFC, $n=726$ ). Para a AFE, foi utilizado o método Robust Diagonally Weighted Least Squares (RDWLS), a partir da matriz de correlação policórica, por meio do software Factor. A AFC e análise dos itens foram realizadas conforme descritas no Estudo 1. Também foram computados os índices Average Variance Extracted (AVE) das escalas original e revisada, que se refere à quantidade de variância explicada pelo construto em relação à variância devido ao erro, por meio do pacote semTools. A fidedignidade dos instrumentos foi verificada por meio do coeficiente alfa de Cronbach e Ômega de McDonald, com o pacote semTools. O escore do participante na escala ENSRA-R foi computado de duas maneiras diferentes: por meio da teoria clássica dos testes (TCT) e da teoria de resposta ao item (TRI). Na TCT, o escore foi obtido a partir do cálculo da média das respostas nos itens; na TRI, o escore se refere aos valores de traço latente dos indivíduos, utilizando método de estimação expected a posteriori, pacote Mirt. Foram, então, realizadas correlações de Pearson entre as variáveis do estudo. Análises fatoriais confirmatórias multigrupo (AFCMG) foram realizadas a fim de verificar a invariância de medida e de médias latentes da ENSRA e da ENSRA-R entre homens e mulheres, utilizando o estimador Maximum Likelihood Robusto (MLR). Enfim, foram realizadas ANOVAs para investigar diferenças sexuais e de orientação sexual nos níveis de satisfação com o relacionamento amoroso. 


\section{Resultados}

Conduziu-se a AFE com os nove itens da ENSRABeta com aproximadamente $50 \%$ da amostra, selecionada aleatoriamente $(n=772)$. Constatou-se adequação dos dados à análise, $K M O=0,93$; Teste de Esfericidade de Barlett, $\chi^{2}(36, N=772)=5296,5 ; p<0,001$. A análise de dimensionalidade pelo método Hull Comparative Fit Index (Lorenzo-Seva et al., 2011) sugeriu a solução unidimensional. O fator explicou 68,3\% da variância comum dos itens. As cargas fatoriais variaram entre 0,65 a 0,94 , exceto para o item 8 (item invertido) que apresentou carga fatorial igual a $-0,48$. As comunalidades dos itens variaram de 0,42 a 0,88 , com exceção do item 8 , que apresentou comunalidade igual a 0,23 .

Diante desse resultado, retirou-se o item 8, pois apresentava carga fatorial menor que 0,50 , como sugerem Comrey \& Lee (1992), e procedeu-se à análise dos parâmetros dos oito itens restantes, pelo modelo GRM, com o total da amostra. Avaliaram-se os parâmetros a e thresholds e optou-se por excluir mais três itens, obtendo-se, assim, a versão revisada da ENSRA (ENSRA-R) com cinco itens. A exclusão dos itens 2 (parâmetro $a=1,36$; thresholds variando de $-2,47$ a 1,27), 5 (parâmetro $a=3,78$; thresholds variando de $-2,38$ a 0,40 ) e 9 (parâmetro $a=2,34$; thresholds variando de $-2,35$ a 0,49 ) ocorreu, pois esses itens apresentavam amplitude de thresholds semelhantes a outros e menores parâmetros a comparados a outros itens. Na Tabela 1, encontram-se os parâmetros dos itens das escalas ENSRA e ENSRA-R. A Figura 1 apresenta a Curva de Informação do Teste ENSRA e do ENSRA-R.
Ainda, testou-se a adequação estrutural do instrumento original (ENSRA, Rusbult et al., 1998) e revisado (ENSRA-R) de maneira confirmatória, com a segunda metade da amostra $(n=726)$. Dado que ambas as versões da escala não seguiam uma distribuição normal multivariada, conforme teste de curtose multivariada de Mardia (ENSRA: $\mathrm{k}=31,7 ; p<0,001$. ENSRA-R: $k=18,83$; $p<0,001)$, o estimador utilizado foi o DWLS.

No que diz respeito ao instrumento original e revisado, observam-se adequados índices de ajustes, indicando que aos dados se ajustam ao modelo unidimensional. Na Tabela 2, encontram-se as cargas fatoriais da ENSRA e ENSRA-R. O AVE da escala original foi igual a 0,67 e da escala revisada, 0,66. Para a ENSRA, os valores dos coeficientes alfa e ômega foram de 0,91. Para ENSRA-R, o valor do coeficiente alfa foi de 0,91 e o ômega de 0,90 .

A fim de obter outras evidências de validade para o instrumento, testaram-se correlações entre diferentes estimações de satisfação com o relacionamento (por meio da ENSRA, ENSRA-R, ENSRA-R-Theta e RelAS), componentes do amor compromisso, intimidade, paixão romântica e paixão erótica (por meio da escala ETA), e questão critério sobre intenção de terminar o relacionamento amoroso. A Tabela 3 mostra os coeficientes de correlação de Pearson entre essas variáveis.

As análises fatoriais confirmatórias multigrupos (AFCMG) indicaram invariância configural, métrica e escalar da ENSRA e da ENSRA-R entre homens e mulheres. Os índices de ajuste obtidos são mostrados na Tabela 4. Além disso, os resultados também indicaram invariância das médias latentes das escalas entre os gêneros.

Tabela 1

Cargas Fatoriais e Parâmetros dos Itens da ENSRA e ENSRA-R

\begin{tabular}{|c|c|c|c|c|c|c|c|c|c|c|}
\hline Item & $\lambda$ & $a$ & $b_{1}$ & $b_{2}$ & $b_{3}$ & $b_{4}$ & $b_{5}$ & $b_{6}$ & $b_{7}$ & $b_{8}$ \\
\hline \multicolumn{11}{|l|}{ ENSRA } \\
\hline 1 & 0,93 & 5,42 & $-2,26$ & $-1,95$ & $-1,66$ & $-1,39$ & $-0,80$ & $-0,56$ & $-0,09$ & 0,51 \\
\hline 2 & 0,52 & 1,31 & $-2,53$ & $-2,25$ & $-1,92$ & $-1,64$ & $-0,33$ & $-0,04$ & 0,49 & 1,29 \\
\hline 3 & 0,86 & 3,13 & $-1,98$ & $-1,74$ & $-1,44$ & $-1,13$ & $-0,47$ & $-0,23$ & 0,29 & 0,88 \\
\hline 4 & 0,91 & 5,29 & $-2,53$ & $-2,14$ & $-1,86$ & $-1,61$ & $-0,98$ & $-0,75$ & $-0,30$ & 0,19 \\
\hline 5 & 0,86 & 3,91 & $-2,39$ & $-2,00$ & $-1,74$ & $-1,47$ & $-0,85$ & $-0,59$ & $-0,13$ & 0,40 \\
\hline \multicolumn{11}{|l|}{ ENSRA-R } \\
\hline 1 & 0,87 & 5,37 & $-2,19$ & $-1,89$ & $-1,62$ & $-1,36$ & $-0,80$ & $-0,57$ & $-0,10$ & 0,50 \\
\hline 3 & 0,88 & 3,35 & $-1,91$ & $-1,68$ & $-1,40$ & $-1,11$ & $-0,47$ & $-0,24$ & 0,28 & 0,87 \\
\hline 4 & 0,82 & 4,49 & $-2,51$ & $-2,11$ & $-1,84$ & $-1,61$ & $-1,00$ & $-0,77$ & $-0,33$ & 0,17 \\
\hline 6 & 0,73 & 2,02 & $-1,09$ & $-0,90$ & $-0,67$ & $-0,46$ & 0,28 & 0,50 & 1,08 & 2,33 \\
\hline 7 & 0,80 & 2,42 & $-1,19$ & $-0,97$ & $-0,67$ & $-0,41$ & 0,23 & 0,44 & 0,92 & 1,83 \\
\hline
\end{tabular}

Nota. Valores dos parâmetros $a$ e $b$ são estandardizados e foram estimados pelo modelo de Resposta Gradual; $b_{1}$ a $b_{8}=i ́ n d i c e ~ d e$ localização ou threshold; $a=$ parâmetro $a$ ou slope; $\lambda=$ carga fatorial estandardizada 

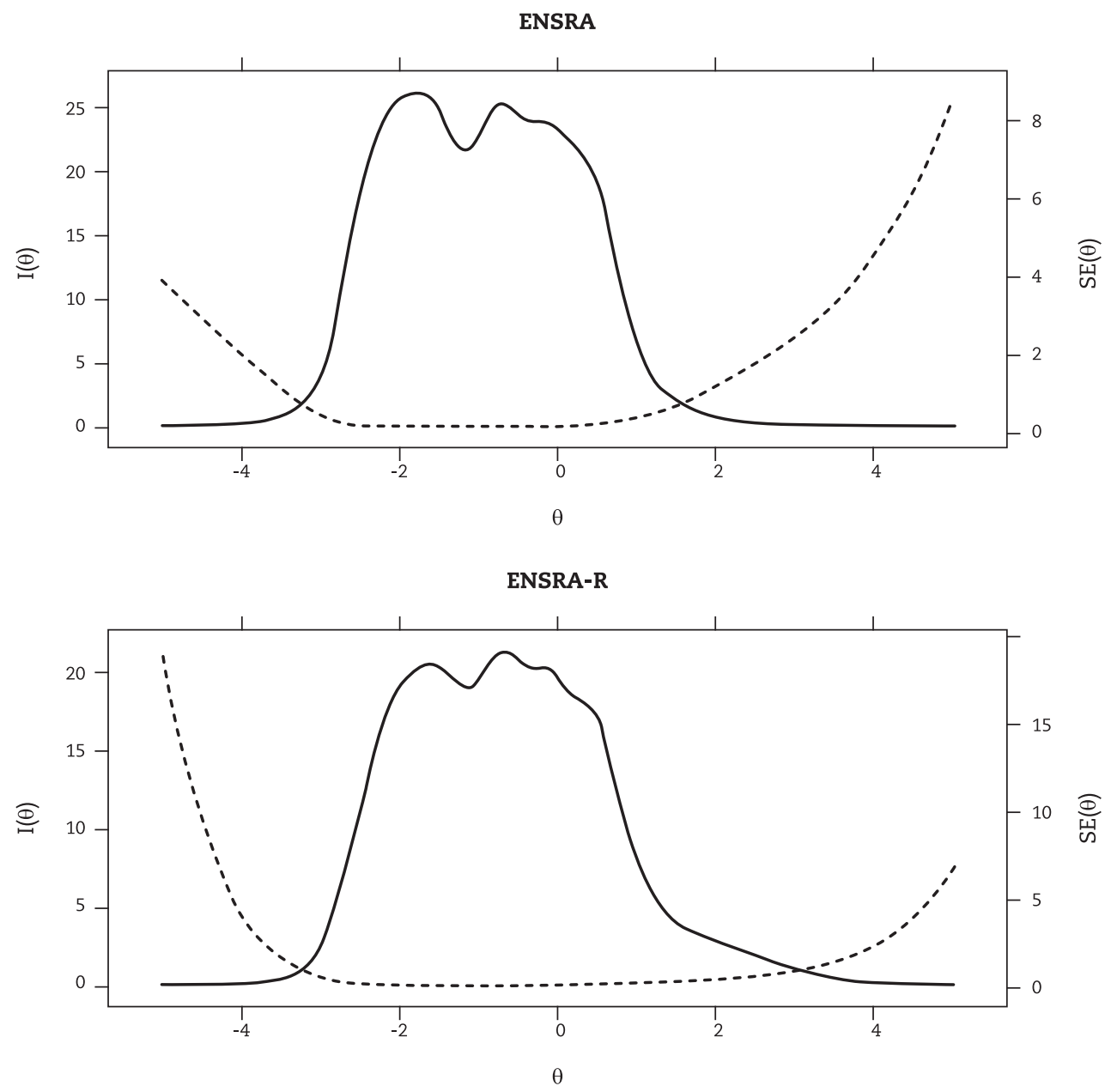

Figura 1. Curvas de Informação dos Testes ENSRA e ENSRA-R, e Erro Padrão

Nota. ENSRA-R (linha contínua) e erro padrão (linha tracejada)

Tabela 2

Índices de Ajuste para os Modelos

\begin{tabular}{lcc}
\hline & & Modelos \\
\cline { 2 - 3 }$\chi^{2}$ & ENSRA & ENSRA-R \\
gl & 6,35 & 20,65 \\
$\mathrm{p}$ & 5 & 5 \\
$\chi^{2} / \mathrm{gl}$ & 0,27 & 0,001 \\
GFI & 1,27 & 4,13 \\
AGFI & 0,998 & 0,996 \\
NFI & 0,994 & 0,988 \\
TLI & 0,996 & 0,991 \\
CFI & 0,998 & 0,992 \\
RMESA & 0,999 & 0,994 \\
IC 90\% RMESA & 0,019 & 0,066 \\
SRMR & $0,000-0,058$ & $0,038-0,096$ \\
\hline
\end{tabular}

Nota. ENSRA=Escala do Nível de Satisfação com o Relacionamento Amoroso; ENSRA-R=Escala do Nível de Satisfação com o Relacionamento Amoroso Revisada; $\chi^{2}=$ qui-quadrado; gl=graus de liberdade; $\chi^{2} / g l=$ razão qui-quadrado por graus de liberdade; GFI=Goodness-of-Fit Index; AGFI=Adjusted Goodness-of Fit Index; NFI=Normed Fit Index; TLI=Tucker-Lewis Index; CFI=Comparative Fit Index; RMESA=Root Mean Square Error of Approximation; IC 90\% RMESA=Intervalo de confiança de 90\%; SRMR=Standardized root mean squared residual 
Tabela 3

Correlações de Pearson entre as Variáveis, Médias, Desvios Padrões, Assimetria e Curtose

\begin{tabular}{|c|c|c|c|c|c|c|c|c|}
\hline & 1 & 2 & 3 & 4 & 5 & 6 & 7 & 8 \\
\hline 1. ENSRA & $(0,91)$ & & & & & & & \\
\hline 2. ENSRA-R & $0,93^{* *}$ & $(0,91)$ & & & & & & \\
\hline 3. ENSRA-R-Theta & $0,92^{* *}$ & $0,96^{* *}$ & & & & & & \\
\hline 4. RelAS & $0,90^{* *}$ & $0,87^{* *}$ & $0,87^{* *}$ & $(0,91)$ & & & & \\
\hline 5. Compromisso & $0,73^{* *}$ & $0,70^{* *}$ & $0,68^{* *}$ & $0,77^{* *}$ & $(0,92)$ & & & \\
\hline 6. Intimidade & $0,77^{* *}$ & $0,76^{* *}$ & $0,75^{* *}$ & $0,79^{* *}$ & $0,69^{* *}$ & $(0,89)$ & & \\
\hline 7. P. Romântica & $0,67^{* *}$ & $0,68^{* *}$ & $0,67^{* *}$ & $0,70^{* *}$ & $0,68^{* *}$ & $0,67^{* *}$ & $(0,85)$ & \\
\hline 8. P. Erótica & $0,42^{* *}$ & $0,44^{* *}$ & $0,44^{* *}$ & $0,47^{* *}$ & $0,45^{* *}$ & $0,42^{* *}$ & $0,67^{* *}$ & $(0,90)$ \\
\hline 9. Freq termin. relac & $-0,73^{* *}$ & $-0,71^{* *}$ & $-0,71^{* *}$ & $-0,78^{* *}$ & $-0,75^{* *}$ & $-0,66^{* *}$ & $-0,59^{* *}$ & $-0,37^{* *}$ \\
\hline M & 5,70 & 4,98 & 0,00 & 5,50 & 4,37 & 4,11 & 3,71 & 3,77 \\
\hline DP & 1,91 & 2,02 & 0,96 & 1,08 & 0,91 & 0,84 & 0,94 & 0,99 \\
\hline Assimetria & $-0,93$ & $-0,52$ & $-0,12$ & $-1,15$ & $-1,70$ & $-1,21$ & $-0,76$ & $-0,84$ \\
\hline Curtose & 0,20 & $-0,59$ & $-0,14$ & 1,26 & 2,32 & 1,21 & 0,02 & 0,14 \\
\hline
\end{tabular}

Nota. ENSRA=Escala do Nível de Satisfação com o Relacionamento Amoroso; ENSRA-R=Escala do Nível de Satisfação com o Relacionamento Amoroso Revisada; ENSRA-R-Theta=Traço latente estimado a partir da ENSRA-R; Compromisso=componente Compromisso da ETA (Escala Tetrangular do Amor); Intimidade=componente intimidade da ETA; P. Romântica=componente Paixão Romântica da ETA; P. Erótica=componente Paixão Erótica da ETA; Freq termin. relac=Frequência de pensar em terminar o relacionamento. Na diagonal, entre parênteses, o coeficiente alfa de Cronbach

Tabela 4

Análises Fatoriais Confirmatórias Multigrupos da ENSRA e ENSRA-R

\begin{tabular}{|c|c|c|c|c|c|}
\hline & & $\chi^{2}\left(\Delta \chi^{2} 2\right)$ & $g l(\Delta g l)$ & $p(\Delta p)$ & CFI $(\triangle \mathrm{CFI})$ \\
\hline \multirow{4}{*}{ ENSRA } & Invariância configural & 117,03 & 10 & $<0,001$ & 0,983 \\
\hline & Invariância métrica & $(3,1041)$ & $(4)$ & $(0,54)$ & $(<0,001)$ \\
\hline & Invariância escalar & $(5,8801)$ & (4) & $(0,21)$ & $(0,001)$ \\
\hline & Invariância das médias & $(0,1096)$ & (1) & $(0,74)$ & $(<0,001)$ \\
\hline \multirow{4}{*}{ ENSRA-R } & Invariância configural & 439,35 & 10 & $<0,001$ & 0,926 \\
\hline & Invariância métrica & $(0,3470)$ & $(4)$ & $(0,99)$ & $(0,001)$ \\
\hline & Invariância escalar & $(2,4426)$ & (4) & $(0,65)$ & $(<0,001)$ \\
\hline & Invariância das médias & $(0,0667)$ & (1) & $(0,80)$ & $(<0,001)$ \\
\hline
\end{tabular}

Nota. $\mathrm{N}=1.498 ;$ gl=grau de liberdade; CFI=Comparative Fit Index

Enfim, foram conduzidas ANOVAs para investigar diferenças de gênero e orientação sexual nas médias de ENSRA e ENSRA-R, bem como possíveis interações entre as variáveis. A interação entre gênero e orientação sexual não foi significativa, tanto para a ENSRA: $F(2,1492)=1,24$; $p=0,24$, quanto para a ENSRA-R: $F(2,1492)=1,11$; $p=0,33$. Também não houve diferenças significativas nem de gênero, ENSRA: $F(1,1492)=0,015 ; p=0,90$; ENSRA-R: $F(1,1492)=0,271 ; p=0,60$, nem de orientação sexual, ENSRA: $F(1,1492)=1,81 ; p=0,29$; ENSRA-R: $F(1,1492)=0,261 ; p=0,77$, em nenhuma das escalas.

\section{Discussão Geral}

O objetivo desta pesquisa foi adaptar e buscar evidências de validade para a população brasileira da Escala do Nível de Satisfação com o Relacionamento Amoroso (Rusbult et al., 1988). Os resultados do primeiro estudo são consistentes com os estudos originais de Rusbult et al. (1998). A análise fatorial confirmatória indicou adequado o modelo unifatorial para explicar os dados provenientes da ENSRA. Os índices de ajuste $\left(\chi^{2}\right.$, CFI, TLI, RMSEA) obtiveram valores dentro do indicado pela literatura (ver Ullman, 2013). A análise dos itens de acordo com a teoria de resposta ao item mostrou que os itens são altamente ou moderadamente discriminativos, conforme proposto por Baker (2001). No entanto, nenhum item do instrumento original apresentou altos valores de parâmetro $b$, isto é, os itens do instrumento original não cobrem altos níveis do traço latente. Essa mesma característica também é encontrada na RelAS (Cassepp-Borges \& Pasquali, 2011). Portanto, o segundo estudo propôs uma 
versão revisada do instrumento, ENSRA-R, incluindo itens com parâmetro $b$ mais elevados.

A ENSRA-R apresentou satisfatórias evidências de validade. Os resultados da análise fatorial exploratória e confirmatória forneceram suporte empírico para um modelo unidimensional reflexivo de satisfação com o relacionamento amoroso. Os índices AVE da escala original e revisada indicaram evidências de validade convergente (Ingoglia, 2013). Os indicadores da ENSRA e ENSRA-R compartilharam, respectivamente, $67 \%$ e $66 \%$ de variância com o construto satisfação com o relacionamento. ENSRA e ENSRA-R mostraram forte correlação positiva com a escala RelAS (Cassepp-Borges \& Pasquali, 2011), sugerindo que todas medem o mesmo construto.

Também se verificaram correlações entre satisfação com o relacionamento e os fatores da escala Tetrangular do Amor (compromisso, intimidade, paixão romântica e paixão erótica). Esse resultado seria esperado, já que pessoas satisfeitas com seus próprios relacionamentos tendem a se sentir conectadas (intimidade), atraídas sexualmente (paixão erótica) e apaixonadas (paixão romântica) pelos seus parceiros, e a se perceber comprometidas com o relacionamento (compromisso). De fato, os resultados desta pesquisa estão em conformidade com achados de pesquisas nacionais e de outros países (e.g., De Andrade et al., 2015; Kochar \& Sharma, 2015; Madey \& Rodgers, 2009; Yela, 2006). Ao mesmo tempo também se esperavam correlações entre satisfação com o relacionamento e a pergunta sobre frequência de pensar em terminar o relacionamento, tal como encontrado em diversos estudos (e.g., Baker et a., 2017; Choice \& Lamke, 1999; Le et al., 2010). Logo, quanto maior a satisfação com o relacionamento amoroso, menor a frequência de pensar em terminar o relacionamento. Isso seria esperado porque, segundo do modelo de investimento do processo de comprometimento (Rusbult et al., 2011), a satisfação é um importante preditor do comprometimento, entendido como intenção em persistir no relacionamento. Em relação à fidedignidade, as escalas ENSRA e ENSRA-R apresentaram adequada consistência interna $(\omega=0,91$ e $\omega=0,90$, respectivamente; $\alpha=0,91$, em ambas as escalas), segundo Pedrabissi e Santinello (1997).

Análises dos itens, por meio do Modelo de Resposta Gradual da TRI (Samejima, 1969), indicou que os itens da ENSRA-R apresentam parâmetros $a$ muito alto (variando de 2,02 a 5,37), conforme Baker (2001). Além disso, avaliando os parâmetros $b$ e inspecionando a curva de informação dos itens da ENSRA-R, observa-se que houve uma ampla cobertura do traço latente satisfação com o relacionamento amoroso (variando de $-2,51$ a 2,33). Logo, pode-se concluir que os cinco itens da ENSRA-R conseguem distinguir eficazmente os indivíduos com diferentes níveis de traço latente. A versão revisada mostra-se mais vantajosa já que pode ser utilizada seja em população clínica (que tende a apresentar baixos níveis de satisfação) seja em população não clínica (que tende a apresentar elevados níveis de satisfação). Ainda, a estimação dos níveis de satisfação com o relacionamento, utilizando a ENSRA-R, deu-se por meio da Teoria Clássica dos Testes e da Teoria de Resposta ao Itens. Pode-se observar que ambas as estimações estão fortemente correlacionadas $(r=0,96)$, indicando que há um compartilhamento de $92,2 \%$ de variância. Assim, é possível concluir que as duas estimações são equivalentes.

As AFCMG indicaram invariância configural, métrica, escalar e de médias da ENSRA e ENSRA-R (Cheung \& Rensvold, 2002). Esses resultados evidenciam que as estruturas de ambas as escalas são estáveis (invariância configural) e que não há vieses de respostas aos instrumentos entre os gêneros. Ainda, a invariâncias das médias evidenciou que, seja na ENSRA, seja na ENSRA-R, as médias latentes de satisfação com o relacionamento amoroso são estatisticamente iguais entre homens e mulheres.

Não foi observado efeito de gênero e de orientação sexual na satisfação com o relacionamento. Indicando que a média dos níveis de satisfação com o relacionamento, em ambas as escalas, são similares entre homens e mulheres e entre heterossexuais, homossexuais e bissexuais. Esses achados vão ao encontro de outros estudos que também não encontraram essas diferenças nos níveis de satisfação com o relacionamento (e.g., Baiocco et al., 2015; Duffy \& Rusbult, 1986; Jackson et al., 2014; Kamp Dush \& Amato, 2005; Rusbult et al., 1998; Ubando, 2016).

As interpretações dos resultados devem considerar as limitações deste estudo. A amostra não probabilística limita inferências e generalizações para a população geral. Sugere-se, ainda, que sejam realizados estudos longitudinais, investigando a relação com outras variáveis e desfechos dos relacionamentos amorosos.

\section{Agradecimentos}

Não há menções.

\section{Financiamento}

Todas as fontes de financiamento para elaboração e produção do estudo (coleta, análise e interpretação dos dados, bem como, escrita dos resultados no presente no manuscrito) foram fornecidas pela bolsa de doutorado do CNPq, pela FAPERJ e pela CAPES - Código de Financiamento 001.

\section{Contribuições dos autores}

Todos os autores participaram da elaboração do manuscrito. Especificamente, o autor Jean Carlos Natividade participou da redação final do trabalho e a autora Terezinha Féres-Carneiro orientou e revisou todos os processos envolvidos na elaboração do artigo. 


\section{Disponibilidade dos dados e materiais}

Todos os dados e sintaxes gerados e analisados durante esta pesquisa serão tratados com total sigilo devido às exigências do Comitê de Ética em Pesquisa em Seres Humanos. Porém, o conjunto de dados e sintaxes que apoiam as conclusões deste artigo estão disponíveis mediante razoável solicitação ao autor principal do estudo.

\section{Conflito de interesses}

Os autores declaram que não há conflitos de interesse.

\section{Referências}

Amirsardari L, \& Khademi A. Psychometric properties of Rusbult's Relationship Investment Scale. Jentashapir Journal of Health Research, 9(6): e85389. https://doi.org/10.5812/jjhr.85389

Baiocco, R., Santamaria, F., Ioverno, S., Fontanesi, L., Baumgartner, E., Laghi, F., \& Lingiardi, V. (2015). Lesbian mother families and gay father families in Italy: Family functioning, dyadic satisfaction, and child well-being. Sexuality Research \& Social Policy: A Journal of the NSRC, 12(3), 202-212. https://doi.org/10.1007/s13178-015-0185-x

Baker, F. B. (2001). The basics of Item Response Theory [Second edition]. Clearinghouse on Assessment and Evaluation, University of Maryland, College Park, MD.

Baker, L. R., McNulty, J. K., \& VanderDrift, L. E (2017). Expectations for future relationship satisfaction: unique sources and critical implications for commitment. Journal of Experimental Psychology: General, 146(5), 700-721. https://doi.org/10.1037/xge0000299

Briñol, P., Petty, R., \& Guyer, J. (2019). A historical view on attitudes and persuasion. Em P. Hegarty, C. Logan, W. Long, P. Pettikainen \& W. Ricken (Eds.), Oxford Encyclopedia of the history of psychology (pp.1-34). New York, NY: Oxford University Press.

Cassepp-Borges, V., \& De Andrade, A. L. (2013). Uma breve história das tentativas para medir atributos dos relacionamentos amorosos em língua portuguesa. Estudos de Psicologia (Natal), 18(4), 631-638. https://doi.org/doi: 10.1590/S1413-294X2013000400011

Cassepp-Borges, V., \& Pasquali, L. (2011). Características psicométricas da relationship assessment scale. Psico-USF, 16(3), 255-264. https:// doi.org/10.1590/ S1413-82712011000300002

Cheung, G. W., \& Rensvold, R. B. (2002). Evaluating goodness-of-fit indexes for testing measurement invariance. Structural Equation Modeling, 9(2), 233-255. https://doi.org/10.1207/S15328007SEM0902_5

Choice, P., \& Lamke, L. K. (1999). Stay/leave decision-making process in abusive dating relationships. Personal Relationships, 6, $351-367$. https://doi.org/10.1111/j.1475-6811.1999.tb00197.x

Comrey, A. L., \& Lee, H. B. (1992). A First Course in Factor Analysis (2nd ed.). Hillsdale, NJ: Lawrence Eribaum Associates.

De Andrade, A. L., \& Garcia, A. (2012). Desenvolvimento de uma medida multidimensional para avaliação de qualidade em relacionamentos românticos-Aquarela-R. Psicologia: Reflexão e Crítica, 25(4), 634-643. https://doi.org/10.1590/S0102-79722012000400002

De Andrade, A. L., Oliveira, M. Z. \& Hatfiel, E. (2017). Conflito trabalho-família: Um estudo com brasileiros e norte-americanos. Revista Psioclogia Organizações e Trabalho, 17(2), 106-113. https://doi.org/10.17652/rpot/2017.2.12738

De Andrade, A. L., Wachelke, J. F. R., \& Howat-Rodrigues, A. B. C. (2015). Relationship satisfaction in young adults: Gender and love dimensions. Interpersona, 9(1), 19-31. https://doi.org/10.5964/ijpr.v9i1.157

Dela Coleta, M. F. (1989). A medida da satisfação conjugal: Adaptação de uma escala. Psico, 18(2), 90-112.

Duffy, S. M., \& Rusbult, C. E. (1986). Satisfaction and commitment in homosexual and heterosexual relationships. Journal of Homosexuality, 12(2), 1-23. https://doi.org/10.1300/J082v12n02_01

França, P. S., Natividade, J. C., \& Lopes, F. A. (2016). Evidências de validade da versão brasileira da escala amor do Marriage and Relationships Questionnaire (MARQ). Psico-USF, 21(2), 233-243. https://doi.org/10.1590/1413-82712016210202

Gable, S. L., \& Poore, J., (2008). Which thoughts count? Algorithms for evaluating satisfaction in relationships. Psychological Science, 19(10), 1030-1036. https://doi.org/10.1111/j.1467-9280.2008.02195.x

Gouveia, V. V., Carvalho, E. A. B., Santos, F. A., \& Almeida, M. R. (2013). Escala Tetrangular do Amor: testando sua estrutura e invariância fatorial. Psicologia: Ciência e Profissão, 33(1), 32-45. https://doi.org/doi: 10.1590/S1414-98932013000100004

Greenwald, A. G., Banaji, M. R., Rudman, L. A., Farnham, S. D., Nosek, B. A., \& Mellott, D. S. (2002). A unified theory of implicit attitudes, stereotypes, self-esteem, and self-concept. Psychological Review, 109(1), 3-25. https://doi.org/10.1037//0033-295X.109.1.3

Greifeneder, R., Bless, H., \& Fiedler, K. (2018). Social cognition (2nd Ed.). New York: Routledge.

Hadden, B. W., \& Knee, C. R. (2018). Finding meaning in us: The role of meaning in life in romantic relationships, The Journal of Positive Psychology, 13(3), 226-239. https://doi.org/10.1080/17439760.2016.1257057

Hendrick, S. S. (1988). A generic measure of relationship satisfaction. Journal of Marriage and the Family, 50(1), 93-98. https://doi. org $/ 10.2307 / 352430$

Hernandez, J. A. E. (2008). Avaliação estrutural da escala de ajustamento diádico, Psicologia em Estudo, 13(3), 593-601. https://doi.org/10.1590/ S1413-73722008000300021

Hernandez, J. A. E. (2014). Evidências de validade da Escala de Avaliação do Relacionamento. Estudos de Psicologia, 21(3), 237-336. https:// doi.org/10.1590/0103-166X2014000300001

Hernandez, J. A. E., Ribeiro, C. M., Carvalho, A. L. N., Fonseca, R. C. T., Peçanha, R. F., \& Falcone, E. M. O. (2017). Revisão da estrutura fatorial da escala de satisfação conjugal. Trends in Psychology, 25(4), 1977-1990. https://doi.org/10.9788/TP2017.4-22Pt

Hoff, C. C., Chakravarty, D., Darbes, L., \& Neilands, T. B. (2019). Studying the motivations behind sexual agreements: A first look at the motivations behind agreement (MBA) scale for male couples. The Journal of Sex Research, 56(6), 718-727. https://doi.org/10.1080/002 24499.2018.1489490

Hollist, C. S., Falceto, O. G., Seibel, B. L., Springer, P. R., Nunes, N. A., Fernandes, C. L. C., \& Miller, R. B. (2016). Depressão pós-parto e satisfação conjugal: Impacto longitudinal em uma amostra brasileira. Revista Brasileira de Medicina de Família e Comunidade. 11(38), 1-13https://doi.org/10.5712/rbmfc11(38)1044 
Impett, E. A., Gable, S. L., \& Peplau, L. A. (2005). Giving up and giving in: The costs and benefits of daily sacrifice in intimate relationships. Interpersonal relations and group processes, 89(3), 327-344. https://doi.org/10.1037/0022-3514.89.3.327

Ingoglia, S. (2013). L'analisi fattoriale confirmative e le sue applicazioni ai problemi della misurazione. Em C. Barbaranelli e S. Ingoglia, I modelli di equazioni strutturali: Temi e prospettive (pp.59-110). Milano: LED.

International Test Commission. (2017). The ITC Guidelines for Translating and Adapting Tests (Second edition). Recuperado de www. InTestCom.org

Jackson, J. B., Miller, R. B., Oka, M., \& Henry, R. G. (2014). Gender differences in marital satisfaction: A meta-analysis. Journal of Marriage and Family, 76(1), 105-129. https://doi.org/10.1111/jomf.12077

Joel, S., Gordon, A. M., Impett, E. A., MacDonald, G., \& Keltner, D. (2013). The things you do for me: Perceptions of a romantic partner's investments promote gratitude and commitment. Personality and Social Psychology Bulletin, 39(10), 1333-1345. https://doi. org $/ 10.1177 / 0146167213497801$

Kamp Dush, C. M., \& Amato, P. R. (2005). Consequences of relationship status and quality for subjective well-being. Journal of Social and Personal Relationships, 22(5), 607-627. https://doi.org/10.1177/0265407505056438

Kochar, R. K., \& Sharma, D. (2015). Role of love in relationship satisfaction. The International Journal of Indian Psychology, 3(1), 80-107. https:// doi.org/10.25215/0301.102

Le, B., Dove, N. L., Agnew, C. R., Korn, M. S., Mutso, A. A. (2010). Predicting nonmarital romantic relationship dissolution: A metaanalytic synthesis. Personal Relationships, 17, 377-390. https://doi.org/10.1111/j.1475-6811.2010.01285.x

Londero-Santos, A., Natividade, J. C., \& Feres-Carneiro, T. (2018). O que faz seu relacionamento ser bom? Associações entre o discurso e a satisfação com o próprio relacionamento. Pôster apresentado na $48^{\mathrm{a}}$ Reunião Anual da Sociedade Brasileira de Psicologia, São Leopoldo, RS.

Londero-Santos, A., Pereira Neto, J. C., \& Natividade, J. C. (2017). Satisfação conjugal e coping diádico como preditores do bem-estar subjetivo. Pôster apresentado na $47^{\mathrm{a}}$ Reunião Anual da Sociedade Brasileira de Psicologia, São Paulo, SP.

Lorenzo-Seva, U., Timmerman, M. E., \& Kiers, H.A.L. (2011). The Hull method for selecting the number of common factors. Multivariate Behavioral Research, 46(2), 340-364. https://doi.org/10.1080/00273171.2011.564527

Madey, S. F., \& Rodgers, L. (2009). The effect of attachment and Sternberg's triangular theory of love on relationship satisfaction. Individual Differences Research, 7(2), 76-84.

McNulty, J. K., Wenner, C. A., \& Fisher, T. D. (2016). Longitudinal associations among relationship satisfaction, sexual satisfaction, and frequency of sex in early marriage. Archives of sexual behavior, 45(1), 85-97. https://doi.org/10,1007/s10508-014-0444-6

Miranda, E. S. (1987). Satisfação conjugal e aspectos relacionados: A influência da comunicação, da semelhança de atitudes e da percepção interpessoal. Arquivos Brasileiros de Psicologia, 39(3), 96-107. http://bibliotecadigital.fgv.br/ojs/index.php/abp/article/view/19749/18473

Müller, B., Nienaber, C. A., Reis, O., Kropp, P., \& Meyer, W. (2014). Sexuality and affection among elderly german men and women in long-term relationships: results of a prospective population-based study. PLOS ONE 9(11): e111404. https://doi.org/10.1371/journal. pone.0111404

Overup, C. S., Hadden, B. W., Knee, C. R., \& Rodriguez, L. M. (2017). Self-determination theory and intimate partner violence (IPV): Assessment of relationship causality orientations as predictors of IPV perpetration. Journal of Research in Personality, 70(2017),139-155. https://doi.org/10.1016/j.jrp.2017.07.002

Pedrabissi, L., \& Santinello, M. (1997). I test psicologici. Bologna: Il Mulino.

Pick de Weiss, S., \& Andrade Palos, P. (1988). Desarrolllo y validacion de la Escala de Satisfaccion Marital. Psiquiatria, 1(4), 9-20. https:// biblat.unam.mx/pt/revista/psiquiatria-mexico-d-f/articulo/desarrollo-y-validacion-de-la-escala-de-satisfaccion-marital

R Development Core Team. (2018). R: A language an environment for statistical computing. R Foundation for Statistical Computing, Vienna, Austria. Software. https://www.R-project.org

Rhatigan, D. L., \& Axsom, D. K. (2006). Using the investment model to understand battered women's commitment to abusive relationships. Journal of Family Violence, 21(2), 153-162. https://doi.org/10.1007/s10896-005-9013-z

Roach, A. J., Frazier, L. P., \& Bowden, S. R. (1981). The marital satisfaction scale: Development of a measure for intervention research. Journal of Marriage and the Family, 43(3), 537. https://doi.org/10.2307/351755

Rodrigues, D., \& Lopes, D. (2013) The Investment Model Scale (IMS): Further studies on construct validation and development of a shorter version (IMS-S), The Journal of General Psychology, 140(1), 16-28. https://doi.org/10.1080/00221309.2012.710276

Rusbult, C. E. (1980). Commitment and satisfaction in romantic associations: A test of the investment model. Journal of Experimental Social Psychology, 16(2), 172-186. https://doi.org/10.1016/0022-1031(80)90007-4

Rusbult, C. E., Agnew, C. R., \& Arriaga, X. B. (2011). The investment model of commitment processes. Department of Psychological Sciences Faculty Publications, 26(2011), 1-33. https://core.ac.uk/download/pdf/19598417.pdf

Rusbult, C. E., Martz, J. M., \& Agnew, C. R. (1998). The investment model scale: Measuring commitment level, satisfaction level, quality of alternatives, and investment size. Personal Relationships, 5(4), 357-387. https://doi.org/10.1111/j.1475-6811.1998.tb00177.x

Samejima, F. (1969). Estimation of latent ability using a response pattern of graded scores. Psychometric Monograph No. 17. Richmond, VA: Psychometric Society.

Scorsolini-Comin, F., \& Santos, M. A. (2011). Ajustamento diádico e satisfação conjugal: Correlações entre os domínios de duas escalas de avaliação da conjugalidade. Psicologia: Reflexão e Crítica, 24(3), 439-447. https://doi.org/10.1590/S0102-79722011000300007

Shackelford, T. K., \& Buss, D. M. (1997). Marital satisfaction in evolutionary psychological perspective. Em R. J. Stenberg \& M. Hojjat (Eds.), Satisfaction in close relationships (pp. 7-25). New York: Guilford.

Sternberg, R. J. (1997). Construct validation of a triangular love scale. European Journal of Social Psychology, 27, 313-335. https://doi.org/10.1002/ (SICI)1099-0992(199705)27:3<313::AID-EJSP824>3.0.CO;2-4

Suntornkanit, N., \& Varma, P. (2018). Influence of gender role attitude, quality of alternatives, investment size, and commitment on marital satisfaction between Thai women who married Thai men and Thai women who married foreign men. Scholar: Human Sciences, 9(2), 106-119. http://www.assumptionjournal.au.edu/index.php/Scholar/article/view/2990/1913

Tan, K., Arriaga, X. B., \& Agnew, C. R. (2018). Running on empty: Measuring psychological dependence in close relationships lacking satisfaction. Journal of Social and Personal Relationships, 35(7), 977-998. https://doi.org/10.1177/0265407517702010

Tran, P., Judge, M., \& Kashima, Y. (2019). Commitment in relationships: An updated meta-analysis of the Investment Model. Personal Relationships, 26(1), 158-180. https://doi.org/10.1111/pere.12268 
Ubando, M. (2016). Gender differences in intimacy, emotional expressivity, and relationship satisfaction. Pepperdine Journal of Communication Research, 4(13), 18-29. https://digitalcommons. pepperdine.edu/cgi/viewcontent. cgi? article=1040\&context=pjcr

Ullman, J. B. (2013). Structural equation modeling. Em B. G. Tabachnick \& L. S. Fidell, Using multivariate statistics (sixth edition, pp. 681 785). New York: Pearson.

VanderDrift, L. E., Agnew, C. R., \& Wilson, J. E. (2014). Spanish version of the investment model scale. Personal Relationships, $21(1), 110-124$. https://doi.org/10.1111/pere.12016

Vecchione, M., Natali, E. M., \& Fida., R. (2013). L'analise di variabili categoriali e non normali. Em C. Barbaranelli \& S. Ingoglia, I modelli di equazioni strutturali (pp. 265-294). Milano: LED.

Wachelke, J. F. R., Andrade, A. L. D., Cruz, R. M., Faggiani, R. B., \& Natividade, J. C. (2004). Medida da satisfação em relacionamento de casal. Psico(USF), 9(1), 11-18. https://doi.org/10.1590/S1413-82712004000100003

Weiser, D. A., \& Weiser, D. J. (2016). Self-efficacy in romantic relationships: Direct and indirect effects on relationship maintenance and satisfaction. Personality and Individual Differences, 89, 152-156. https://doi.org/10.1016/j.paid.2015.10.013

Wickham, R. E. (2013). Perceived authenticity in romantic partners. Journal of Experimental Social Psychology, 49(5), 878-887. https://doi. org/10.1016/j.jesp.2013.04.001

Wickham, R. E., Reed, D. E., \& Williamson, R. E. (2015). Establishing the psychometric properties of the Self and Perceived-partner Authenticity in Relationships Scale-Short Form (AIRS-SF): Measurement invariance, reliability, and incremental validity. Personality and Individual Differences, 77(2015), 62-67. https://doi.org/10.1016/j.paid.2014.12.049

Winking, J., Eastwick, P. W., Smith, L. K., \& Koster, J. (2018). Applicability of the Investment Model Scale in a natural-fertility population. Personal Relationships, 25(4), 497-516. https://doi.org/10.1111/pere.12257

Yela, C. (2006). The evaluation of love: Simplified version of the scales for Yela's tetrangular model based on Sternberg's model. European Journal of Psychological Assessment, 22(1), 21-27. https://doi.org/10.1027/1015-5759.22.1.21

\section{Sobre os autores}

Amanda Londero-Santos é psicóloga e mestre pela Università degli Studi di Padova (Itália) e doutora em Psicologia (PUC-Rio). Atualmente é professora do Departamento de Psicometria da UFRJ.

Jean Carlos Natividade é professor do Programa de Pós-Graduação em Psicologia da PUC-Rio.

Terezinha Féres-Carneiro é psicóloga (PUC-Rio), mestre (PUC-Rio) e doutora em Psicologia Clínica (PUC-SP) e tem pósdoutorado em Psicoterapia de Casal e Família (Universidade Paris Descartes). Atualmente é professora titular da PUC-Rio.

\section{Como citar este artigo}

Londero-Santos, A., Natividade, J. C., \& Féres-Carneiro, T. (2021). Uma Medida de Satisfação com o Relacionamento Amoroso. Avaliação Psicológica, 20(1), 11-22. http://dx.doi.org/10.15689/ap.2020.2001.18901.02 